Article

\title{
Visualization of the Effect of Assay Size on the Error Profile of Tumor Mutational Burden Measurement
}

\author{
Nathanael G. Bailey (D)
}

check for

updates

Citation: Bailey, N.G. Visualization of the Effect of Assay Size on the Error Profile of Tumor Mutational Burden Measurement. Genes 2022, 13, 432. https://doi.org/10.3390/ genes13030432

Academic Editors: Pawel Mroz, Bryan L. Betz and Charles Billington

Received: 8 February 2022

Accepted: 24 February 2022

Published: 26 February 2022

Publisher's Note: MDPI stays neutral with regard to jurisdictional claims in published maps and institutional affiliations.

Copyright: (C) 2022 by the author. Licensee MDPI, Basel, Switzerland. This article is an open access article distributed under the terms and conditions of the Creative Commons Attribution (CC BY) license (https:// creativecommons.org/licenses/by/ $4.0 /)$.
Department of Pathology, University of Pittsburgh and UPMC, Pittsburgh, PA 15213, USA; baileyng@upmc.edu

\begin{abstract}
Tumor mutational burden (TMB) refers to the number of somatic mutations in a tumor per megabase and is a biomarker for response to immune checkpoint inhibitor therapy. Immune checkpoint inhibitors are currently approved for tumors with TMB greater than or equal to 10 mutations/megabase. Many laboratories are currently reporting TMB values based upon targeted resequencing panels with limited genomic coverage. Due to sampling variation, this leads to significant uncertainty in the assay's TMB result, particularly at relatively low TMB levels near the 10 mutation per megabase therapeutic threshold. In order to allow clinicians and laboratorians to explore this uncertainty, we built a novel web application that allows a user to view the potential error of a TMB result given the sequencing panel size. This application also allows the user to explore the effect of incorporating knowledge of a specific tumor type's typical TMB distribution on the error profile of the TMB result.
\end{abstract}

Keywords: tumor mutation burden; immune checkpoint inhibitors; precision medicine

\section{Introduction}

Tumor mutational burden (TMB) is defined by the number of somatic mutations present in a tumor exome. TMB is an emerging therapeutic biomarker that is associated with response to immune checkpoint inhibitors (ICI): tumors with higher TMB exhibit better responses to ICIs. This is thought to be due to increased immunogenicity of tumors with high TMB values due to the accumulation of neoantigens [1]. Thresholds ranging from 10 mutations/megabase (mut/Mb) to 20 mut/Mb have been proposed to dichotomize tumors into TMB-low and TMB-high categories [2,3]. Recently, pembrolizumab was approved by the FDA for use in tumors with TMB values of 10 mut/Mb or greater, irrespective of histology [4,5]; this decision has been the subject of much debate [6-9].

Although TMB is defined as the exomic mutation count, few tumors currently have whole-exome sequencing performed. Instead, mutations are enumerated in targeted sequencing assays that generally sequence around $1 \mathrm{Mb}$ (approximately $1 / 30$ th of the entire exome). The mutation number is then divided by the total $\mathrm{Mb}$ sequenced to determine TMB. Importantly, these assays were initially designed primarily to assess for known hotspot mutations in oncogenes and for mutations in tumor suppressor genes, and there is no reason to think that the number of mutations in the genes included in the targeted panel is of any more biologic importance for neoantigen formation and ICI response than are mutations of unsequenced genes. Therefore, the validity of the TMB result given by the assay is directly related to its ability to predict the "true," unmeasured TMB. This ability is dependent on the sampling error of the assay, which is related to the panel size of the sequencing assay. Specifically, if it is assumed that mutations are essentially independent and identically distributed, then the assay's mutation count is distributed binomially given the number of bases sequenced and the underlying probability of mutation at each base. It stands to reason that sequencing more bases and identifying more mutations improves the error profile of a TMB assay, and it has been well recognized that TMB accuracy is dependent upon the assay panel size [3,10-15]. However, the implication of this uncertainty 
for an individual patient's TMB result may be difficult for a clinician to visualize. We set out to make an application that displays TMB measurement uncertainty given user-defined inputs of a TMB value and the panel size of the sequencing assay.

\section{Materials and Methods}

An R Shiny web application was written that accepts a target TMB value and the assay size in megabases as user inputs. Based on the entered assay size, the application calculates the assay-specific number of mutations that would need to be detected to most closely generate the entered target TMB value. The probability mass function of the mutation count given the panel size and the underlying true mutation frequency is then modeled with the binomial distribution: mutation count $\sim$ Binomial (bases sequenced, probability of mutation at each base).

The probability distribution of the true TMB value given the calculated assay-specific mutation count is determined using the dbinom $\mathrm{R}$ function, the user-selected size of the assay, and a vector of "true" mutational probabilities equivalent to $0-200 \mathrm{mut} / \mathrm{Mb}$, in increments of 0.1. The resulting vector of probabilities given a TMB assay result is then normalized and plotted as the likelihood distribution, and it is next sampled to generate information such as the central $90 \%$ confidence interval and the likelihood that the "true" $\mathrm{TMB}$ value is greater than $10 \mathrm{mut} / \mathrm{Mb}$.

The application additionally draws on publicly available mutational data for patients treated with ICI from cBioportal $[16,17]$ and previously reported by Samstein et al. [18]. This dataset contains TMB values for 1661 patients with various tumor histologies. The user may elect to display histology-specific TMB deciles to visualize the uncertainty of a TMB percentile assignment to an individual case. The $R$ function bkde is used to generate an empiric binned kernel density estimate based on this public mutational data to produce histology-specific TMB prior distributions. At the user's discretion, the app can incorporate this prior information regarding histology-specific TMB distributions to determine the impact of this knowledge on the measured TMB result using Bayes's theorem, plotting the prior distribution, the likelihood distribution, and the posterior distribution that takes the prior histology-specific TMB distribution information into account.

The application was built with R v.4.1.0 and packages shiny v.1.7.1 and KernSmooth $v$.2.23-20. A version of the $R$ script suitable for running on a local $R$ installation using RStudio is contained in the supplemental files. The working app is available at https:/ / pathology.shinyapps.io/tmbapp/ (accessed on 7 February 2022).

\section{Results}

\subsection{Illustration of the Application Interface}

The user selects a target TMB value, the size of the sequencing assay, and whether to use an informative prior distribution. The application displays the density function of the TMB result, along with the middle $90 \%$ confidence interval (CI), the corresponding percentile range, and the probability that the true TMB result is $>10 \mathrm{mut} / \mathrm{Mb}$ as seen in Figure 1. 


\section{Effect of Sequencing Assay Size on Uncertainty of TMB Calculation}
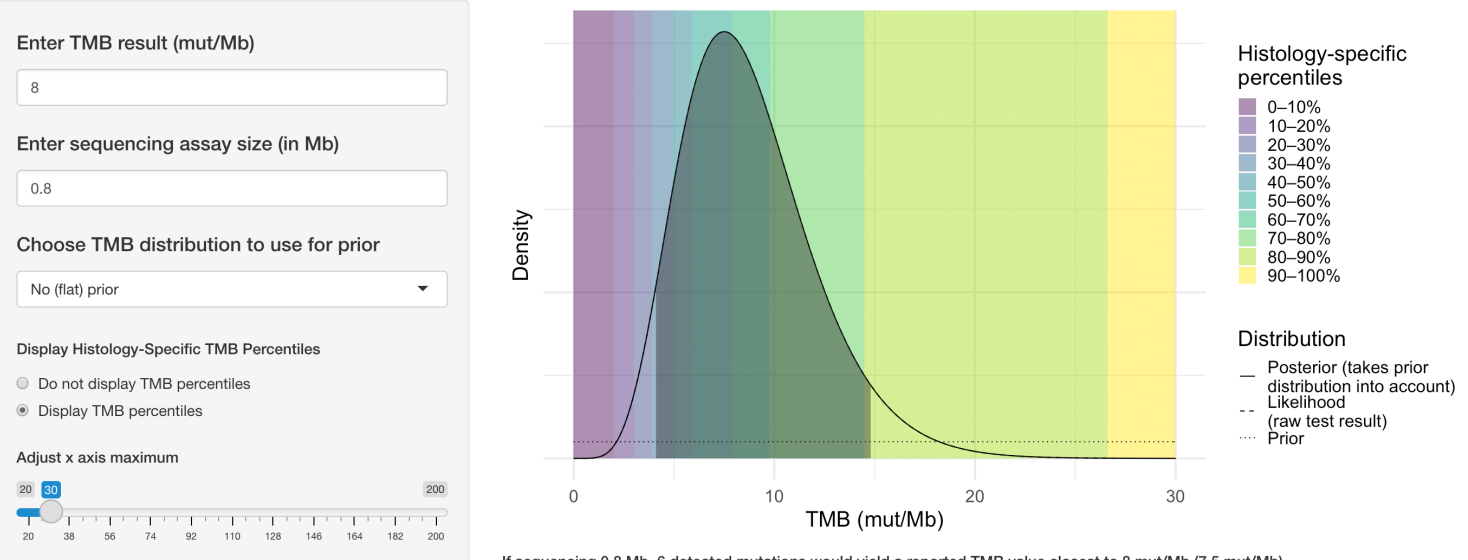

Without considering prior information about the distribution of TMB, the middle $90 \%$ confidence interval for this result ranges from 4.1 to
14.9 mut/Mb (shaded region in plot). The chance that the true TMB is greater than 10 mut $/ \mathrm{Mb}$ is $31 \%$. For all tumors, this corresponds to a percentile range of $33 \%$ to $81 \%$.

Figure 1. Example of the web application interface. The user selects the target TMB result, the sequencing assay size in megabases, whether to use prior information regarding the TMB distribution for the tumor type, and whether to display TMB percentile information. The application then displays the central $90 \%$ confidence interval for the true TMB result and calculates the likelihood that the true result is greater than 10 mutations per megabase.

\subsection{Effect of Assay Size on Uncertainty}

Figure 2 illustrates the significant effect of assay size on the confidence interval and corresponding percentile range for a TMB result of $8 \mathrm{mut} / \mathrm{Mb}$.
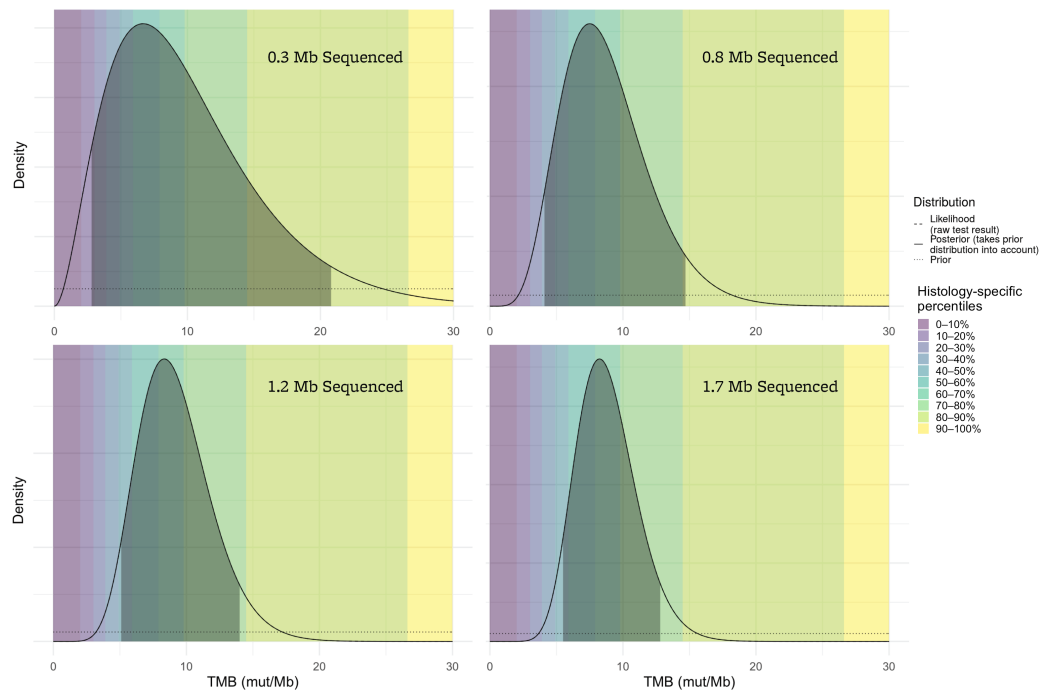

Figure 2. The accuracy of the TMB result is highly dependent upon the number of megabases sequenced. If very few megabases are sequenced (top left panel), the range of plausible true TMB values (indicated by the shaded areas of the plots) given the assay result is very broad, while results from larger sequencing assays (such as the bottom right) are more reliable.

\subsection{Significant Assay Uncertainty at Low TMB Values}

If approximately $0.8 \mathrm{Mb}$ is sequenced, such as in the FDA-approved companion diagnostic test for pembrolizumab, a TMB-low result of 5 mut/Mb could actually reflect a true TMB value of $>10$ mut/Mb approximately $10 \%$ of the time, as seen in Figure 3 . 

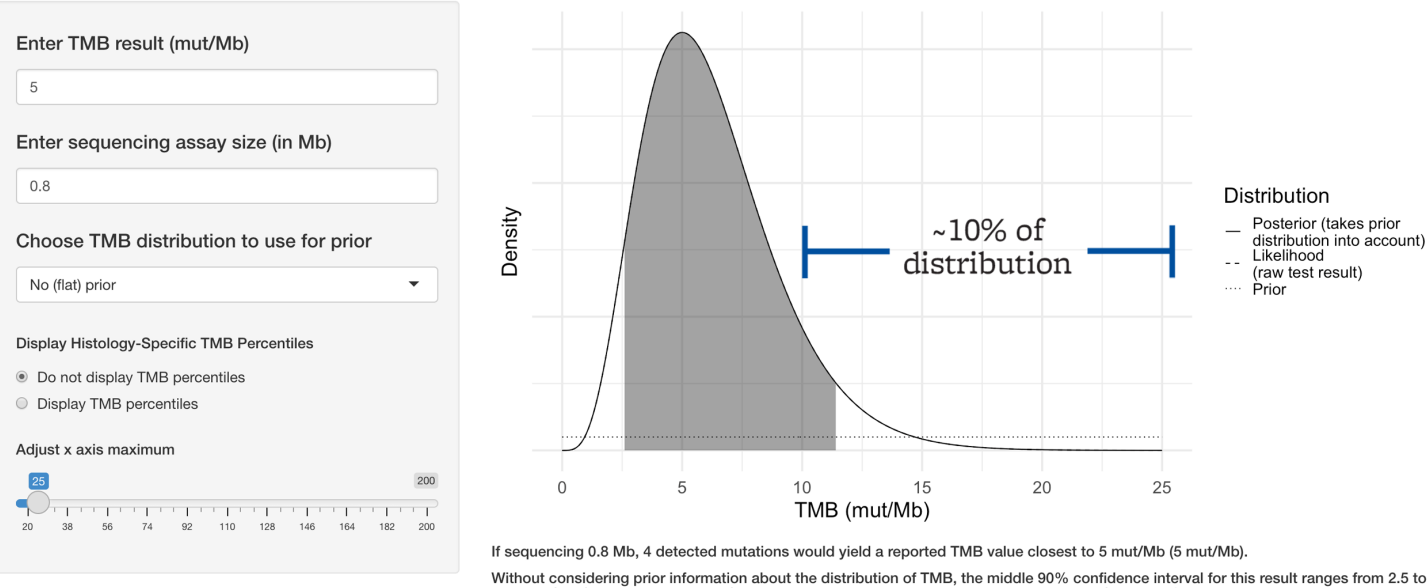

Without considering prior information about the distribution of TMB, the middle $90 \%$ confidence interval
$11.5 \mathrm{mut} / \mathrm{Mb}$ (shaded region in plot). The chance that the true $\mathrm{TMB}$ is greater than $10 \mathrm{mut} / \mathrm{Mb}$ is $10 \%$.

Figure 3. The threshold for clinical response to immune checkpoint inhibitor therapy is $10 \mathrm{mut} / \mathrm{Mb}$ based on TMB alone. FDA-approved companion diagnostic TMB assays sequence approximately $0.8 \mathrm{Mb}$. Even a common TMB-low value such as $5 \mathrm{mut} / \mathrm{Mb}$ generated by such an assay would indicate a true $\mathrm{TMB}$ value of greater than 10 mut/ $\mathrm{Mb}$ approximately $10 \%$ of the time.

\subsection{Effect of Incorporating Knowledge of Prior Distribution}

If significantly less than $1 \mathrm{Mb}$ is sequenced, the confidence interval of TMB results is highly dependent upon the tumor-specific prior distribution.

If only $0.3 \mathrm{Mb}$ is sequenced, a TMB value of 12 mut/Mb would suggest that there is an $81 \%$ chance that the true TMB is greater than 10 mut/Mb, assuming a flat prior (Figure 4, left panel). However, most tumors have TMB values less than $10 \mathrm{mut} / \mathrm{Mb}$, and incorporating that a priori information will tend to decrease the range of plausible values for the "true" TMB result. Different tumors have different TMB distributions, and this effect can greatly impact the implications of a TMB result if small sequencing panels such as this are used. For example, with an assay that is $0.3 \mathrm{Mb}$ in size, if the sample is melanoma (with a high pretest probability of increased $\mathrm{TMB}$ ), the probability that an assay result of 12 mut/ $\mathrm{Mb}$ indicates a true TMB result of $>10$ mut/ $\mathrm{Mb}$ is $68 \%$ (Figure 4 , center). However, if the sample is a renal cell carcinoma (where TMB is expected to be low), the probability is only $43 \%$ (Figure 4 , right panel).
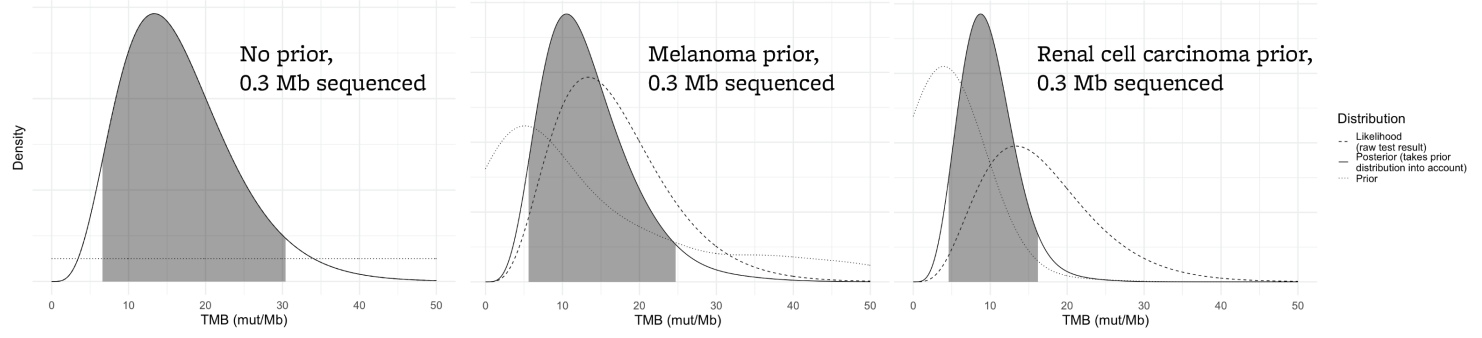

Figure 4. For a small assay sequencing $0.3 \mathrm{Mb}$, the confidence interval of a TMB result is affected by the tumor type that is being sequenced. Each plot assumes an assay result of $12 \mathrm{mut} / \mathrm{Mb}$. The left panel demonstrates the likely true TMB result (gray area) without taking prior knowledge of tumor TMB distributions into account. The true TMB result will likely be higher in a tumor type that tends to have higher TMB values (such as melanoma, center plot) versus a tumor type that tends to have lower TMB values (renal cell carcinoma, right plot).

As demonstrated in Figure 5, if $1.7 \mathrm{Mb}$ is sequenced, the posterior distribution of a $\mathrm{TMB}$ result of $12 \mathrm{mut} / \mathrm{Mb}$ is far less dependent on the prior because the test result itself is much more informative (likelihood that the true TMB is greater than 10 mut/Mb: flat prior $79 \%$, melanoma $73 \%$, renal cell carcinoma $63 \%$ ). 


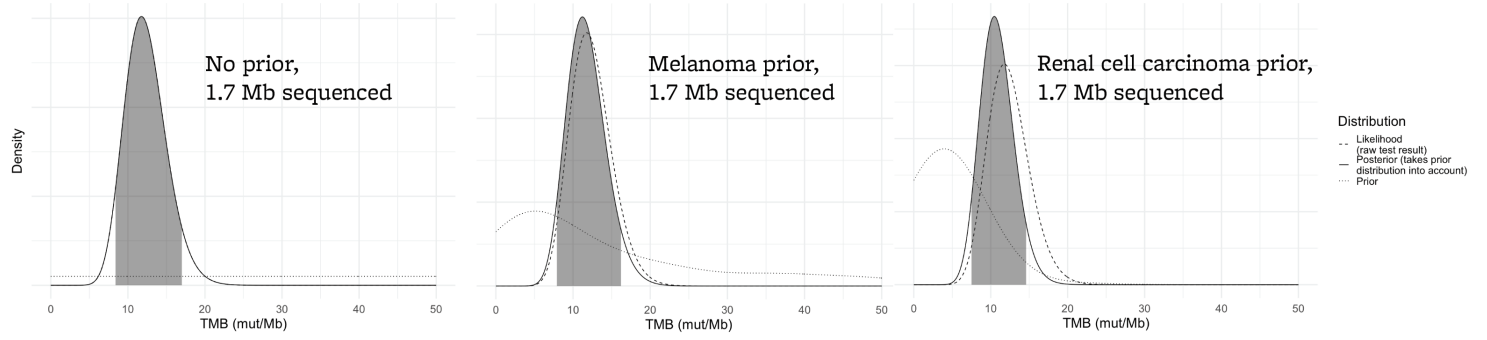

Figure 5. For a larger assay sequencing $1.7 \mathrm{Mb}$, the effect of the prior distribution on the "true" TMB result is less marked. All panels assume an assay-generated TMB value of 12 mut/Mb.

\subsection{Very High TMB Values Are Less Problematic}

Although significant uncertainty remains with regard to the true TMB value, even small targeted sequencing panels are adequate to classify a tumor as TMB-high if many mutations are identified, as seen in Figure 6.
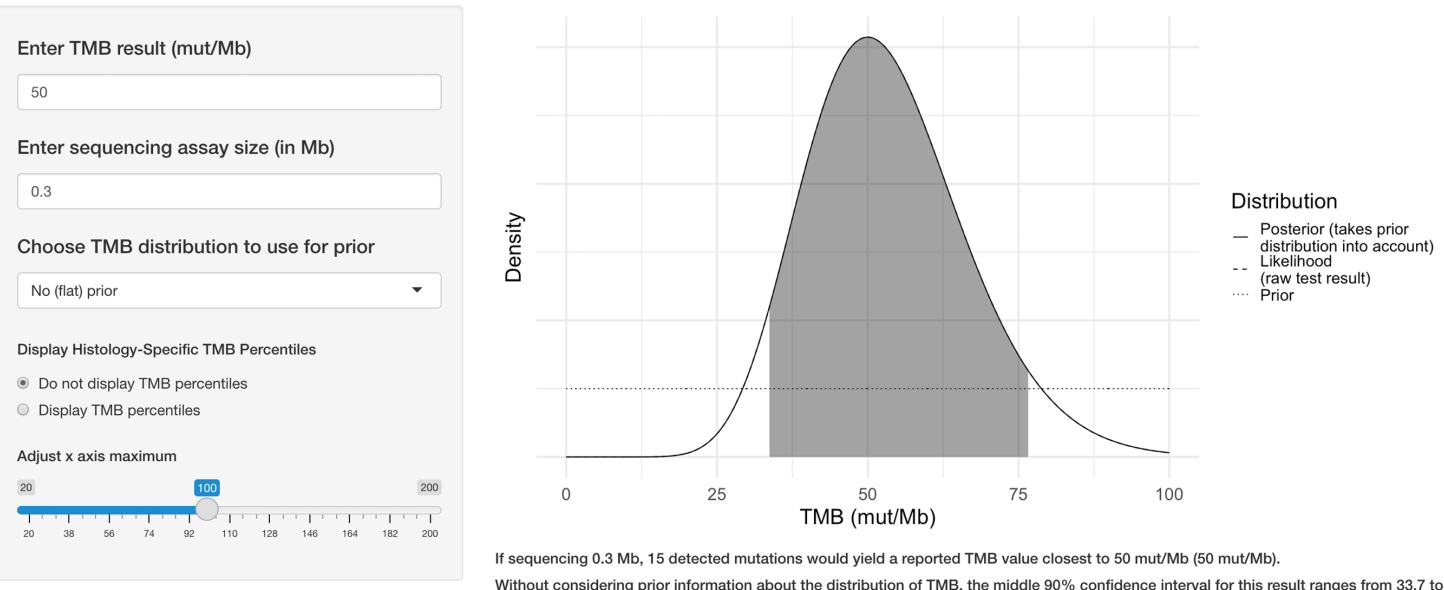

If sequencing $0.3 \mathrm{Mb}, 15$ detected mutations would yield a reported TMB value closest to $50 \mathrm{mut} / \mathrm{Mb}$ (50 mut/Mb).

Without considering prior information about the distribution of TMB, the middle $90 \%$ confidence interval for this result ranges from 33.7 to

Figure 6. If many mutations are identified far from the 10 mut/Mb threshold, even very small panels can reliably establish that a tumor is TMB-high with confidence.

\section{Discussion}

The web application provides a user-friendly and convenient way to explore the uncertainty of a TMB result due to sampling error. There are several limitations of this application. Sources of TMB measurement error other than those derived from sampling such as variant allele fraction thresholds, distinction between germline and somatic variants (which may be particularly challenging for patients from under-represented groups [19]), and potential targeted panel bias toward hotspot mutations are not reflected in the output of the application. These additional sources of error are likely substantial when using small panels, where each incremental mutation identified has a large effect on the TMB calculation. This application also makes some simplifying assumptions regarding the distribution of mutations through the exome, as tumors contain mutational signatures reflecting their underlying mutational processes, and the distributions of mutations throughout the exome are not entirely random [20]. These sources of assay uncertainty are not reflected in the simple binomial model used in this application, and the output of the application should be considered a first approximation of the assay error.

Clinicians are familiar with the concept that some laboratory results need to be interpreted in conjunction with the pre-test probability of a condition: for many assays, clinicians are trained to consider an assay's sensitivity and specificity in light of a condition's prevalence to determine the more clinically meaningful positive and negative predictive values for the assay result. Ideally, analytical laboratory assays would be suf- 
ficiently robust such that the pre-test prior distribution of possible results is relatively unimportant when interpreting the results of the assay, but this is not the case with TMB results as currently measured. The prior distribution of TMB values for a given tumor type can significantly impact the plausible "true" TMB value, just as the prevalence of a disease can markedly impact the predictive value of an assay with a given sensitivity and specificity. Incorporating prior knowledge about the histology-specific TMB distribution can lead to a more accurate estimation of TMB, particularly if very small sequencing panels are used. Since most tumors have TMB values $<10$ mut/ $\mathrm{Mb}$, incorporating this knowledge typically decreases the plausible TMB range. For example, a TMB-high result is more likely to be "true" if a melanoma is being sequenced than if a renal cell carcinoma is being sequenced, unless the assay is of sufficient size to mitigate the uncertainty due to sampling error or unless a sufficiently high number of mutations are identified.

Studies have shown good correlation between TMB determined by targeted sequencing panels and ICI response [4,18], but sampling errors as modeled in this application would normalize across a large cohort of patients; showing that an assay correlates with an outcome using cohort-level metrics has limited implications for an individual patient's result if it cannot be accurately measured. In a cohort of patients with TMB measured by small panels, some patients' TMB values will be overestimated and others will be underestimated, still leading to an positive overall association between TMB and ICI response, as the measurement errors will tend to cancel one another out and the overall trend will remain. However, in routine clinical practice, the question is not whether an assay's TMB results correlate with ICI response across a cohort of patients; rather, it is "can the assay accurately determine the TMB-high/low status and therefore therapy for this individual patient?" Unfortunately, currently used small sequencing panels cannot definitively answer this question for the great majority of patient tumors with TMB values that are below or near the TMB-high threshold of 10 mut/Mb, as has been recognized in ongoing TMB standardization efforts [14]. TMB is likely a predictor for ICI response as a continuous variable, reflecting increasing neoantigen density in a tumor. While any threshold used for dichotomization of a continuous variable is intrinsically arbitrary and difficult to justify [21], the current FDA-approved threshold for TMB is especially problematic, as a patient's TMB cannot be measured with confidence near the 10 mut/Mb threshold with many currently used assays. However, for highly mutated tumors, such as those with DNA mismatch repair deficiency or POLE/POLD1 mutations (also populations where the benefit of ICI is most clear $[9,22-24])$, the clinical performance of assays with small panel sizes may be clinically adequate, as the plausible interval for the true TMB value will be unlikely to contain the TMB-high threshold in spite of the increasing variation in the actual numerical TMB result generated by the assay [14].

\section{Conclusions}

The application provides a convenient way to approximate the error intrinsic to TMB measurement by targeted resequencing approaches. Laboratories reporting TMB should consider acknowledging the uncertainty of TMB results in their reports, particularly near clinically relevant thresholds. Assays that sequence significantly less than $1 \mathrm{Mb}$ should not be used to determine TMB. Ongoing standardization efforts will remain important, along with additional studies to better determine biomarkers that are most predictive of ICI response.

Supplementary Materials: The following supporting information can be downloaded at: https: / / www.mdpi.com/article/10.3390/genes13030432/s1, File: app.R, which provides code sufficient to run the application on a local $\mathrm{R}$ installation.

Funding: This research received no external funding.

Institutional Review Board Statement: Not applicable.

Informed Consent Statement: Not applicable. 
Data Availability Statement: The R code for the web application is available as a supplemental file.

Acknowledgments: The author would like to thank Kelly M. Bailey, M.D., for reading the manuscript and providing constructive comments.

Conflicts of Interest: The authors declare no conflict of interest.

Abbreviations
The following abbreviations are used in th
$\begin{array}{ll}\mathrm{TMB} & \text { Tumor mutational burden } \\ \mathrm{ICI} & \text { Immune checkpoint inhibitor } \\ \text { mut/Mb } & \text { Mutations per megabase }\end{array}$

\section{References}

1. Rizvi, N.A.; Hellmann, M.D.; Snyder, A.; Kvistborg, P.; Makarov, V.; Havel, J.J.; Lee, W.; Yuan, J.; Wong, P.; Ho, T.S.; et al. Cancer immunology. Mutational landscape determines sensitivity to PD-1 blockade in non-small cell lung cancer. Science 2015, 348, 124-128. [CrossRef]

2. Goodman, A.M.; Kato, S.; Bazhenova, L.; Patel, S.P.; Frampton, G.M.; Miller, V.; Stephens, P.J.; Daniels, G.A.; Kurzrock, R. Tumor Mutational Burden as an Independent Predictor of Response to Immunotherapy in Diverse Cancers. Mol. Cancer Ther. 2017, 16, 2598-2608. [CrossRef] [PubMed]

3. Chalmers, Z.R.; Connelly, C.F.; Fabrizio, D.; Gay, L.; Ali, S.M.; Ennis, R.; Schrock, A.; Campbell, B.; Shlien, A.; Chmielecki, J.; et al. Analysis of 100,000 human cancer genomes reveals the landscape of tumor mutational burden. Genome Med. 2017, 9, 34. [CrossRef]

4. $\quad$ Marabelle, A.; Fakih, M.; Lopez, J.; Shah, M.; Shapira-Frommer, R.; Nakagawa, K.; Chung, H.C.; Kindler, H.L.; Lopez-Martin, J.A.; Miller, W.H.; et al. Association of tumour mutational burden with outcomes in patients with advanced solid tumours treated with pembrolizumab: Prospective biomarker analysis of the multicohort, open-label, phase 2 KEYNOTE-158 study. Lancet Oncol. 2020, 21, 1353-1365. [CrossRef]

5. Marcus, L.; Fashoyin-Aje, L.A.; Donoghue, M.; Yuan, M.; Rodriguez, L.; Gallagher, P.S.; Philip, R.; Ghosh, S.; Theoret, M.R.; Beaver, J.A.; et al. FDA Approval Summary: Pembrolizumab for the Treatment of Tumor Mutational Burden-High Solid Tumors. Clin. Cancer Res. 2021, 27, 4685-4689. [CrossRef]

6. Prasad, V.; Addeo, A. The FDA approval of pembrolizumab for patients with TMB >10 mut/Mb: Was it a wise decision? No. Ann. Oncol. 2020, 31, 1112-1114. [CrossRef]

7. $\quad$ McGrail, D.J.; Pilié, P.G.; Rashid, N.U.; Voorwerk, L.; Slagter, M.; Kok, M.; Jonasch, E.; Khasraw, M.; Heimberger, A.B.; Lim, B.; et al. High tumor mutation burden fails to predict immune checkpoint blockade response across all cancer types. Ann. Oncol. 2021, 32, 661-672. [CrossRef] [PubMed]

8. Valero, C.; Lee, M.; Hoen, D.; Zehir, A.; Berger, M.F.; Seshan, V.E.; Chan, T.A.; Morris, L.G.T. Response Rates to Anti-PD-1 Immunotherapy in Microsatellite-Stable Solid Tumors With 10 or More Mutations per Megabase. JAMA Oncol. 2021, 7, 739-743. [CrossRef]

9. Rousseau, B.; Foote, M.B.; Maron, S.B.; Diplas, B.H.; Lu, S.; Argilés, G.; Cercek, A.; Diaz, L.A., Jr. The Spectrum of Benefit from Checkpoint Blockade in Hypermutated Tumors. N. Engl. J. Med. 2021, 384, 1168-1170. [CrossRef] [PubMed]

10. Garofalo, A.; Sholl, L.; Reardon, B.; Taylor-Weiner, A.; Amin-Mansour, A.; Miao, D.; Liu, D.; Oliver, N.; MacConaill, L.; Ducar, M.; et al. The impact of tumor profiling approaches and genomic data strategies for cancer precision medicine. Genome Med. 2016, 8, 79. [CrossRef]

11. Endris, V.; Buchhalter, I.; Allgäuer, M.; Rempel, E.; Lier, A.; Volckmar, A.L.; Kirchner, M.; von Winterfeld, M.; Leichsenring, J.; Neumann, O.; et al. Measurement of tumor mutational burden (TMB) in routine molecular diagnostics: In silico and real-life analysis of three larger gene panels. Int. J. Cancer 2019, 144, 2303-2312. [CrossRef] [PubMed]

12. Budczies, J.; Allgäuer, M.; Litchfield, K.; Rempel, E.; Christopoulos, P.; Kazdal, D.; Endris, V.; Thomas, M.; Fröhling, S.; Peters, S.; et al. Optimizing panel-based tumor mutational burden (TMB) measurement. Ann. Oncol. 2019, 30, 1496-1506. [CrossRef] [PubMed]

13. Buchhalter, I.; Rempel, E.; Endris, V.; Allgäuer, M.; Neumann, O.; Volckmar, A.L.; Kirchner, M.; Leichsenring, J.; Lier, A.; von Winterfeld, M.; et al. Size matters: Dissecting key parameters for panel-based tumor mutational burden analysis. Int. J. Cancer 2019, 144, 848-858. [CrossRef] [PubMed]

14. Merino, D.M.; McShane, L.M.; Fabrizio, D.; Funari, V.; Chen, S.J.; White, J.R.; Wenz, P.; Baden, J.; Barrett, J.C.; Chaudhary, R.; et al. Establishing guidelines to harmonize tumor mutational burden (TMB): in silico assessment of variation in TMB quantification across diagnostic platforms: Phase I of the Friends of Cancer Research TMB Harmonization Project. J. Immunother. Cancer 2020, 8. [CrossRef] [PubMed]

15. Budczies, J.; Kazdal, D.; Allgäuer, M.; Christopoulos, P.; Rempel, E.; Pfarr, N.; Weichert, W.; Fröhling, S.; Thomas, M.; Peters, S.; et al. Quantifying potential confounders of panel-based tumor mutational burden (TMB) measurement. Lung Cancer 2020, 142, 114-119. [CrossRef] [PubMed] 
16. Cerami, E.; Gao, J.; Dogrusoz, U.; Gross, B.E.; Sumer, S.O.; Aksoy, B.A.; Jacobsen, A.; Byrne, C.J.; Heuer, M.L.; Larsson, E.; et al. The cBio cancer genomics portal: An open platform for exploring multidimensional cancer genomics data. Cancer Discov. 2012, 2, 401-404. [CrossRef]

17. Gao, J.; Aksoy, B.A.; Dogrusoz, U.; Dresdner, G.; Gross, B.; Sumer, S.O.; Sun, Y.; Jacobsen, A.; Sinha, R.; Larsson, E.; et al. Integrative analysis of complex cancer genomics and clinical profiles using the cBioPortal. Sci. Signal. 2013, 6, pl1. [CrossRef] [PubMed]

18. Samstein, R.M.; Lee, C.H.; Shoushtari, A.N.; Hellmann, M.D.; Shen, R.; Janjigian, Y.Y.; Barron, D.A.; Zehir, A.; Jordan, E.J.; Omuro, A.; et al. Tumor mutational load predicts survival after immunotherapy across multiple cancer types. Nat. Genet. 2019, 51, 202-206. [CrossRef]

19. Asmann, Y.W.; Parikh, K.; Bergsagel, P.L.; Dong, H.; Adjei, A.A.; Borad, M.J.; Mansfield, A.S. Inflation of tumor mutation burden by tumor-only sequencing in under-represented groups. NPJ Precis. Oncol. 2021, 5, 22. [CrossRef]

20. Alexandrov, L.B.; Kim, J.; Haradhvala, N.J.; Huang, M.N.; Tian Ng, A.W.; Wu, Y.; Boot, A.; Covington, K.R.; Gordenin, D.A.; Bergstrom, E.N.; et al. The repertoire of mutational signatures in human cancer. Nature 2020, 578, 94-101. [CrossRef]

21. Altman, D.G.; Royston, P. The cost of dichotomising continuous variables. BMJ 2006, 332, 1080. [CrossRef] [PubMed]

22. Le, D.T.; Durham, J.N.; Smith, K.N.; Wang, H.; Bartlett, B.R.; Aulakh, L.K.; Lu, S.; Kemberling, H.; Wilt, C.; Luber, B.S.; et al. Mismatch repair deficiency predicts response of solid tumors to PD-1 blockade. Science 2017, 357, 409-413. [CrossRef]

23. Wang, F.; Zhao, Q.; Wang, Y.N.; Jin, Y.; He, M.M.; Liu, Z.X.; Xu, R.H. Evaluation of POLE and POLD1 Mutations as Biomarkers for Immunotherapy Outcomes Across Multiple Cancer Types. JAMA Oncol. 2019, 5, 1504-1506. [CrossRef] [PubMed]

24. Rousseau, B.J.C.; Bieche, I.; Pasmant, E.; Simmet, V.; Hamzaoui, N.; Masliah-Planchon, J.; Pouessel, D.; Bruyas, A.; Augereau, P.; Grob, J.J.; et al. $526 \mathrm{O}$ High activity of nivolumab in patients with pathogenic exonucleasic domain POLE (edPOLE) mutated Mismatch Repair proficient (MMRp) advanced tumours. Ann. Oncol. 2020, 31, S463. [CrossRef] 